\title{
A $C^{2}$-continuous rational quintic interpolation scheme for curve data with shape control
}

\author{
Malik Zawwar Hussain ${ }^{1}$, Maria Hussain ${ }^{2 *}$ and Zahra Yameen ${ }^{1}$ \\ ${ }^{1}$ Department of Mathematics, University of the Punjab, Lahore, Pakistan. \\ ${ }^{2}$ Lahore College for Women University, Lahore, Pakistan.
}

\begin{abstract}
A new rational quintic interpolation scheme for curve design is presented. The new scheme is suggested for scalar 2D data and has three free parameters that provide design flexibility. Constraints are developed on one of the free parameters to preserve the shape of positive and range restricted data. The other two parameters are free to guarantee design flexibility. The resulting rational quintic interpolation scheme is globally $C^{2}$-continuous and its order of continuity is independent of the choice of free parameters and data values. Approximation order of the proposed interpolation scheme is three. Application of the $C^{2}$-continuous rational quintic interpolation scheme and the role of free parameters are demonstrated by several examples.
\end{abstract}

Keywords: Approximation order, $C^{2}$-continuity, free parameters, positive and range restricted data, rational quintic function.

\section{INTRODUCTION}

Cubic spline is the lowest degree piecewise $C^{2}$-continuous curve and it avoids Runge's phenomenon (Schumaker, 2007). However, cubic spline imposes constraints on first order derivatives at knots to ensure $C^{2}$-continuity at those knots. Moreover, alteration of derivative value at one knot effects the derivative values globally over the whole domain. Keeping in view the above mentioned limitation of cubic spline interpolation, a $C^{2}$-continuous piecewise rational quintic interpolation scheme is introduced in this paper for 2D data. The interpolation scheme is local and variation of derivative values in one subinterval does not affect the derivative values in other intervals. Although the degree of presented rational interpolant is five, the undesired oscillations of the interpolated curve can be controlled by sensibly choosing the values of free parameters. The role of free parameters is demonstrated by developing numerical schemes for the shape preservation of hereditary shapes of data like positivity and range restriction. In many physically existing phenomena, quantities have a meaning only if their values are positive. The quantity of scums of different toxic substances in rivers is always positive (Hussain, 2006). Medication's diffusion rate in blood, durability of radioactive material (Hussain et al., 2014), probability, population statistics, rainfall amount etc., are some entities, which are always positive.

The interpolation schemes that are meant for the preservation of inherited shapes of data are known as shape-preserving interpolation schemes. Positivity, monotonicity and convexity are the inherited shapes of data. Let $\left\{\left(f_{i}, d_{i}, D_{i}\right): i=0,1,2, \ldots, n\right\}$ be the $2 \mathrm{D}$ data defined over the interval $\left[x_{0}, x_{n}\right]$ with ordinate values $f_{i}$; first and second order derivative values $d_{i}$ and $D_{i}$ at knots $x_{i}, \forall i$. Given dataset is convex if $d_{i}<d_{i+1}$ and $D_{i}>0, \forall i$. The same dataset is monotone increasing if $f_{i}<f_{i+1}$ and $d_{i}>0, \forall i$, and positive if $f_{i}>0, \forall i$.

The problem of shape-preserving interpolation was discussed by various researchers (Schmidt \& Hess, 1988; Goodman et al., 1991; Butt \& Brodlie, 1993; Lamberti \& Manni, 2001; Wang \& Tan, 2004; Duan et al., 2005; Yong-juan \& Guo-jin, 2007; Hussain \& Sarfraz, 2008; Hussain et al., 2011; 2014; Sarfraz et al., 2013; Lettieri

\footnotetext{
* Corresponding author (maria.hussain@lcwu.edu.pk; iD https://orcid.org/0000-0002-4403-8997)
} 
et al., 2014). Cubic curves (Schmidt \& Hess, 1988; Butt \& Brodlie, 1993; Lamberti \& Manni, 2001), trigonometric curves (Yong-juan \& Guo-jin, 2007; Hussain et al., 2014) and rational cubic functions (Schmidt \& Hess, 1988; Hussain \& Sarfraz, 2008; Hussain et al., 2011; Sarfraz et al., 2013) were used for developing shape-preserving schemes.

Butt and Bordlie (1993) used cubic Hermite interpolant for shape preservation of $2 \mathrm{D}$ positive data for given values of derivatives at knots. The algorithm worked in two steps: first, those intervals were identified where the interpolated curve lost positivity; then the identified intervals were broken down into subintervals by inserting one or more knots. Goodman et al. (1991) presented two schemes for the graphical display of data subject to linear constraints. The first scheme adopted the method of scaling weights and the second scheme refers to inserting some extra points to preserve the shape of data. Hussain et al. (2009) proposed a piecewise $C^{2}$ - rational quintic function with two free parameters for the interpolation of curve data. The effect of free parameters on the shape of the curve was illustrated through numerical results. It was observed that for very large and small values of free parameters the $C^{2}$ rational quintic function reduced to the non-rational polynomial curve. Hussain et al. (2014) devised a $G C^{1}$ quadratic trigonometric interpolant with two parameters for the shape preservation of curve data. Lamberti and Manni (2013) used parametric cubic Hermite interpolant and derived constraints on subinterval's length to preserve data heredity. First order derivatives at knots were estimated by a tri-diagonal system of equations. The parametric cubic Hermite interpolant produced $C^{2}$-continuous curve for these estimated values of derivatives. In Lettieri et al. (2014), $C^{3}$ and $C^{4}$ shapepreserving interpolation schemes were introduced. These interpolation schemes were based on two parameter family of rational quintic function. The interpolant was completely determined by solving a linear sparse system. The shape parameters were locally determined to preserve the shape of data locally. Schmidt and Hess (1988) derived sufficient conditions on derivatives at the knots to preserve the positivity of interpolating cubic polynomial. This technique was restricted for data without derivatives. Yong-juan and Guo-jin (2007) used Bézier like trigonometric curve in control point form for shape preservation of convex curve data. The trigonometric curve (Yong-juan \& Guo-jin, 2007) had linear basis functions and one free parameter $\alpha$. The range of free parameter was specified for convexity preservation of curve data. In Hussain and Sarfraz (2008) and Sarfraz et al. (2013), the authors devised $C^{1}$-rational interpolation schemes for shape preservation of curve data. The shape parameters were constrained to preserve the shape of curve data. In Duan et al. (2005), the authors assumed suitable values of parameters to obtain $C^{2}$-continuous curve and the method worked for equally spaced data. In Hussain et al. (2011), a shape-preserving $C^{2}$-rational interpolation scheme was introduced to guarantee the shape preservation of positive and convex curve data. Here, first order derivatives were found by a system of linear equations. Only for these values of derivatives of rational interpolation scheme Hussain et al. (2011) assured $C^{2}$-continuity at knots.

In this research paper, a $C^{2}$-rational quintic interpolation scheme is introduced for curve data with three free parameters. Shape-preserving constraints are derived on these free parameters to construct $C^{2}$-shapepreserving rational interpolation schemes. A comparison of the proposed schemes to the existing ones is detailed as follows:

- In Duan et al. (2005), the $C^{2}$ shape-preserving rational interpolation scheme worked for equally spaced data. The $C^{2}$ shape-preserving scheme proposed in this research paper is applicable to uniform as well as nonuniform data.

- In some previous studies (Goodman et al., 1991; Butt \& Brodlie, 1993; Lamberti \& Manni, 2001), the authors attained the desired shape of the data by inserting extra knots in the challenging subinterval. The numerical scheme proposed in this research paper does not refine the domain.

- In Hussain et al. (2009), a $C^{2}$-rational quintic interpolating function with two free parameters was introduced. The rational quintic interpolating function introduced in this research paper has three parameters. Thus it has more degrees of freedom than the interpolating function (Hussain et al., 2009). The rational quintic interpolant (Hussain et al., 2009) was unable to preserve the inherited shapes of data while in this research paper, the numerical schemes are formulated for shape preservation of positive and constrained 2D data. The approximation order of both above mentioned numerical schemes is three but the value of optimal error coefficient $\left(C_{i}\right)$ differs. In Hussain et al. (2009), $C_{i}=0.0335$ whilst for $C^{2}$-rational quintic interpolating function constructed in the present study, $C_{i}=0.0219$. It follows that the error of interpolation scheme proposed in this research paper is less than the interpolation scheme in Hussain et al. (2009). Thus it is computationally efficient than Hussain et al. (2009).

- Shape-preserving rational interpolation schemes used in Hussain and Sarfraz (2008) and Sarfraz et al. (2013) were $C^{1}$ whereas in this research paper a $C^{2}$ interpolation scheme is devised for shape preservation of curve data. 
- In Hussain et al. (2011), the $C^{2}$-continuous shapepreserving interpolation schemes were achieved by imposing constraints on first order derivatives at knots. The $C^{2}$-rational quintic interpolation scheme proposed in this research paper interpolates first as well as second order derivatives at knots. Hence, unlike Hussain et al. (2011) it is applicable to data, as well as data regarding derivatives. The value of optimal error coefficient for Hussain et al. (2011) is $C_{i}=8 / 3$ which is enormous. Hence the $C^{2}$-rational quintic interpolating scheme proposed in this research paper is more efficient than Hussain et al. (2011).

- In Lettieri et al. (2014), the interpolant was completely determined by solving a linear sparse system of equations. The proposed $C^{2}$-rational quintic function is completely determined by the functional values, first and second order derivatives at knots and there is no need to solve a system of equations. Therefore, the use of $C^{2}$-rational quintic interpolation scheme proposed in this research paper requires less computer memory than the interpolation scheme introduced in Lettieri et al. (2014).

\section{METHODOLOGY}

In this research paper, a $C^{2}$-rational quintic function with three free parameters is introduced and its convergence is analysed. Further, the problem of shape-preserving interpolation is dealt.

\section{$C^{2}$-rational quintic function}

The piecewise rational functions are in use for interpolation of scalar data (Hussain \& Sarfraz, 2008; Sarfraz et al., 2013). Although these rational interpolation schemes are local, the order of continuity of these schemes at knots is limited to $C^{1}$-continuity. In Duan et al. (2005) and Hussain et al. (2011), the order of continuity of rational interpolation schemes was increased by applying $C^{2}$-continuity at knots. It resulted in a tri-diagonal and diagonally dominant system of equations in unknown first order derivatives at knots. Therefore, the interpolation schemes (Duan et al., 2005; Hussain et al., 2011) were not suitable for interpolation of data with first order derivatives, e.g. for interpolation of solutions of ordinary differential equations. Moreover, the number of unknown first order derivatives at knots was greater than the number of equations yielded by imposing $C^{2}$-continuity at knots. Variation of the value of the first order derivative at one knot affects the derivative values at the remaining knots. Hence the $C^{2}$ rational interpolation schemes proposed in Duan et al. (2005) and Hussain et al. (2011) were global. Due to the above mentioned shortfalls of $C^{2}$-rational interpolation schemes (Duan et al., 2005; Hussain et al., 2011), a novel $C^{2}$-rational interpolation function is introduced here.

Let $x_{i}<x_{i+1}, i=0,1,2, \ldots, n-1$, be the partition of interval $[a, b]$ with $x_{0}=a$ and $x_{n}=b$. Take $f_{i}, d_{i}$ and $D_{i}$ as functional values, first and second derivative values at knots $x_{i}, i=0,1,2, \ldots, n$. The proposed $C^{2}$-rational quintic function (RQF), $S(x)$, is defined over each subinterval as:

$S(x) \equiv S_{i}(x)=\frac{p_{i}(\theta)}{q_{i}(\theta)}$.

Here,

$p_{i}(\theta)=\sum_{j=0}^{5}(1-\theta)^{5-j} \theta^{j} A_{j}$

$A_{0}=\alpha_{i} f_{i}, A_{1}=\left(3 \alpha_{i}+\beta_{i}\right) f_{i}+\alpha_{i} h_{i} d_{i}$,

$A_{2}=\left(3 \alpha_{i}+3 \beta_{i}+\gamma_{i}\right) f_{i}+\left(2 \alpha_{i}+\beta_{i}\right) h_{i} d_{i}+0.5 \alpha_{i} h_{i}^{2} D_{i}$,

$A_{3}=\left(3 \gamma_{i}+3 \beta_{i}+\alpha_{i}\right) f_{i+1}-\left(2 \gamma_{i}+\beta_{i}\right) h_{i} d_{i+1}+0.5 \gamma_{i} h_{i}^{2} D_{i+1}$,

$A_{4}=\left(3 \gamma_{i}+\beta_{i}\right) f_{i+1}-\gamma_{i} h_{i} d_{i+1}, A_{5}=\gamma_{i} f_{i+1}$,

$q_{i}(\theta)=\alpha_{i}(1-\theta)^{2}+\beta_{i}(1-\theta) \theta+\gamma_{i} \theta^{2}$,

$\theta=\frac{x-x_{i}}{h_{i}}, h_{i}=x_{i+1}-x_{i}, i=0,1,2 \ldots, n-1$.

Here $\theta$ is the normalised variable, i.e. $\theta \in[0,1]$. The RQF defined in equation (1) has three positive real free parameters $\left(\alpha_{i}>0, \beta_{i}>0, \gamma_{i}>0\right)$. These free parameters can produce a family of curves for the given data by modifying the values of free parameters. RQF satisfies the following interpolation conditions:

$$
\begin{gathered}
S\left(x_{i}\right)=f_{i}, S\left(x_{i+1}\right)=f_{i+1}, S^{\prime}\left(x_{i}\right)=d_{i}, S^{\prime}\left(x_{i+1}\right)=d_{i+1}, \\
S^{\prime \prime}\left(x_{i}\right)=D_{i}, S^{\prime \prime}\left(x_{i+1}\right)=D_{i+1} .
\end{gathered}
$$

It follows from (2) that the rational quintic function (1) is a $C^{2}$-continuous function (interpolates data, first and second order derivatives) at knots. If $\alpha_{i}=1, \beta_{i}=2$ and $\gamma_{i}=1$, the RQF becomes quintic Hermite interpolant (Farin, 2005). In the rest of this research paper, $\alpha_{i}, \beta_{i}$, and $\gamma_{i}$ are assumed to be positive real free parameters.

\section{Arithmetic mean approximation scheme for derivatives}

The given 2D data $\left\{\left(x_{i}, f_{i}\right): i=0,1,2, \ldots, n\right\}$ defined over the interval $[a, b]$, where $x_{i}<x_{i+1}, i=0,1,2, \ldots, n-1$. The arithmetic mean choice of first order derivatives $d_{i}$ at knots $x_{i}$ are given by the following formula: 
$d_{0}=\Delta_{0}+\left(\Delta_{0}-\Delta_{1}\right) h_{0}\left(h_{0}+h_{1}\right)^{-1} ;$

$d_{n}=\Delta_{n-1}+\left(\Delta_{n-1}-\Delta_{n-2}\right) h_{n-1}\left(h_{n-1}+h_{n-2}\right)^{-1} ;$

$d_{i}=0.5\left(\Delta_{i}+\Delta_{i-1}\right) ; i=1,2,3, \ldots, n-1$.

Here, $\Delta_{i}=\frac{f_{i+1}-f_{i}}{h_{i}}, h_{i}=x_{i+1}-x_{i}, i=0,1,2, \ldots, n-1$.

Similarly the second order derivatives $D_{i}$ at knots $x_{i}$ are given by the following formula:

$D_{0}=M_{0}+\left(M_{0}-M_{1}\right) h_{0}\left(h_{0}+h_{1}\right)^{-1}$;

$D_{n}=M_{n-1}+\left(M_{n-1}-M_{n-2}\right) h_{n-1}\left(h_{n-1}+h_{n-2}\right)^{-1}$;

$D_{i}=0.5\left(M_{i}+M_{i-1}\right) ; i=1,2,3, \ldots, n-1$.

Here, $M_{i}=\frac{d_{i+1}-d_{i}}{h_{i}}, h_{i}=x_{i+1}-x_{i}, i=0,1,2, \ldots, n-1$.

\section{Some observations}

Here, some observations for the effect of free parameters on the shape of curve are shared mathematically and graphically.

(1) Interval tension: The $C^{2}$-rational quintic function (1) is rewritten as follows:

$S(x) \equiv S_{i}(x)=(1-\theta) f_{i}+\theta f_{i+1}+\frac{N(\theta)}{q_{i}(\theta)}$,

$N(\theta)=(1-\theta)^{4} \theta\left\{\alpha_{i}\left(f_{i}-f_{i+1}\right)+\alpha_{i} h_{i} d_{i}\right\}+(1-\theta)^{3} \theta^{2}\left\{\left(2 \alpha_{i}+\beta_{i}\right)\right.$

$\left.\left(f_{i}-f_{i+1}\right)+\left(2 \alpha_{i}+\beta_{i}\right) h_{i} d_{i}+0.5 \alpha_{i} h_{i}^{2} D_{i}\right\}+(1-\theta)^{2} \theta^{3}\left\{\left(2 \gamma_{i}+\beta_{i}\right)\right.$

$\left.\left(f_{i+1}-f_{i}\right)-\left(2 \gamma_{i}+\beta_{i}\right) h_{i} d_{i+1}+0.5 \gamma_{i} h_{i}^{2} D_{i+1}\right\}+$

$(1-\theta) \theta^{4}\left\{\gamma_{i}\left(f_{i+1}-f_{i}\right)-\gamma_{i} h_{i} d_{i+1}\right\}$

From equation (5), the following observation is made:

$$
\begin{aligned}
\lim _{\alpha_{i} \rightarrow \infty, \beta_{i} \rightarrow \infty} S_{i}(x) & =\lim _{\alpha_{i} \rightarrow \infty, \gamma_{i} \rightarrow \infty} S_{i}(x)=\lim _{\gamma_{i} \rightarrow \infty, \beta_{i} \rightarrow \infty} S_{i}(x) \\
& =(1-\theta) f_{i}+\theta f_{i+1} .
\end{aligned}
$$

It is noted that by simultaneously increasing either of the two free parameters in the interval $I_{i}=\left[x_{i}, x_{i+1}\right]$, the $C^{2}$-rational quintic function (1) reduces to a straight line $(1-\theta) f_{i}+\theta f_{i+1}$. This phenomenon is known as interval tension behaviour of $C^{2}$-rational quintic function (1).
(2) From equation (1), following observations are made:

$$
\begin{aligned}
& \lim _{\alpha_{i} \rightarrow 0, \beta_{i} \rightarrow 0} S_{i}(x)=(1-\theta)^{3} f_{i}+(1-\theta)^{2} \theta\left\{3 f_{i+1}-2 h_{i} d_{i+1}+\right. \\
& \left.0.5 h_{i}^{2} D_{i+1}\right\}+(1-\theta) \theta^{2}\left\{3 f_{i+1}-h_{i} d_{i+1}\right\}+\theta^{3} f_{i+1} . \\
& \lim _{\gamma_{i} \rightarrow 0, \beta_{i} \rightarrow 0} S_{i}(x)=(1-\theta)^{3} f_{i}+(1-\theta)^{2} \theta\left\{3 f_{i}+h_{i} d_{i}\right\}+
\end{aligned}
$$

$(1-\theta) \theta^{2}\left\{3 f_{i}+2 h_{i} d_{i}+0.5 h_{i}^{2} D_{i}\right\}+\theta^{3} f_{i+1}$.

$\lim _{\gamma_{i} \rightarrow 0, \alpha_{i} \rightarrow 0} S_{i}(x)=(1-\theta)^{3} f_{i}+(1-\theta)^{2} \theta\left\{3 f_{i}+h_{i} d_{i}\right\}+$

$(1-\theta) \theta^{2}\left\{3 f_{i+1}-h_{i} d_{i+1}\right\}+\theta^{3} f_{i+1}$.

(3) The data in Table 1 is generated from constant function $f(x)=3$ over the domain $[0,4]$. The partition of this interval is $\{0,1.5,3,3.2,4\}$. The constant data in Table 1 is interpolated by the $C^{2}$-continuous rational quintic function (1) for different values of free parameters $\alpha_{i}, \beta_{i}$ and $\gamma_{i}$ (Figures 1 and 2). Graphical results suggest that the $C^{2}$-continuous rational quintic function (1) interpolate the constant data in the desired shape (staright line) for the random choice of free parameters.

Table 1: Data points generated from constant function

\begin{tabular}{llllll}
\hline$x$ & 0 & 1.5 & 3 & 3.2 & 4 \\
$f$ & 3 & 3 & 3 & 3 & 3 \\
\hline
\end{tabular}

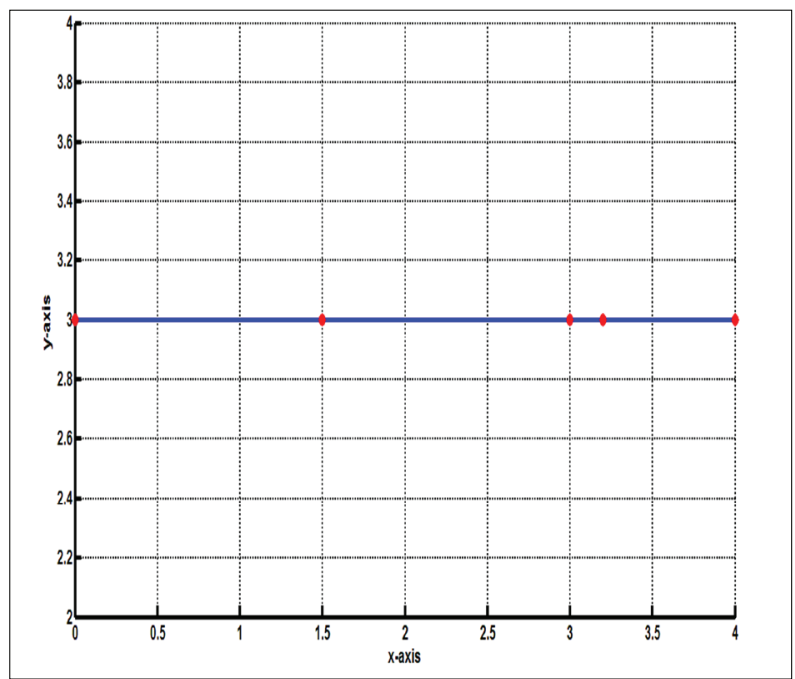

Figure 1: $C^{2}$-rational quintic interpolation of constant function with $\alpha_{i}=0.0300, \beta_{i}=3, \gamma_{i}=0.0300, \forall i$. 


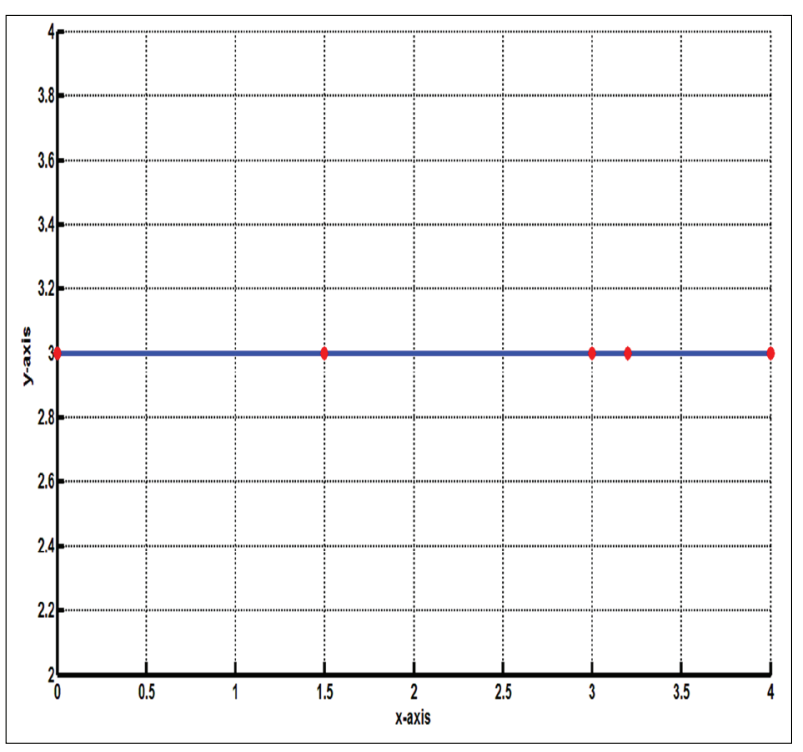

Figure 2: $C^{2}$-rational quintic interpolation of constant function with $\alpha_{i}=10, \beta_{i}=6, \gamma_{i}=15, \forall i$

(4) If $\alpha_{i}=0, \beta_{i}=1, \gamma_{i}=0$, the RQF (1) becomes cubic Hermite interpolant (Farin, 2005).

(5) Given the 2D data $\left\{\left(f_{i}, d_{i}, D_{i}\right): i=0,1,2, \ldots, n\right\}$ defined over the interval $\left[x_{0}, x_{n}\right]$, if we choose $\alpha_{i}=0, \beta_{i}=1, \gamma_{i}=0$ in each subinterval and apply the second order derivative continuity condition at interior knots $x_{i}, i=1,2,3, \ldots, n-1$, then RQF (1) is a cubic spline interpolant on $\left[x_{0}, x_{n}\right]$.

(6) Given the 2D data $\left\{\left(f_{i}, d_{i}, D_{i}\right): i=0,1,2, \ldots, n\right\}$ defined over the interval $\left[x_{0}, x_{n}\right]$ if we choose $\alpha_{i}=1, \beta_{i}=2, \gamma_{i}=1$ in each subinterval and apply the third and fourth order derivative continuity condition at interior knots $x_{i}, i=1,2,3, \ldots, n-1$ then RQF (1) is a quintic spline interpolant on $\left[x_{0}, x_{n}\right]$. It is already mentioned that for $\alpha_{i}=1, \beta_{i}=2, \gamma_{i}=1$, the RQF (1) becomes quintic Hermite interpolant.

Theorem 1. Absolute error of interpolation of $C^{2}$-rational quintic function $S(x)$, defined in equation (1), for $f(x) \in C^{3}\left[x_{0}, x_{n}\right]$, satisfies the following relation

Error $=|f(x)-S(x)| \leq\left\|f^{(3)}\right\| h_{i}^{3} c_{i}$,

with optimal error coefficient $=c_{i}=\max _{0 \leq \theta \leq 1} w\left(\alpha_{i}, \beta_{i}, \gamma_{i}, \theta\right)$.

Here, $w\left(\alpha_{i}, \beta_{i}, \gamma_{i}, \theta\right)=w_{1}\left(\alpha_{i}, \beta_{i}, \gamma_{i}, \theta\right)$ for $0 \leq \theta \leq \theta^{*}$

and $w\left(\alpha_{i}, \beta_{i}, \gamma_{i}, \theta\right)=w_{2}\left(\alpha_{i}, \beta_{i}, \gamma_{i}, \theta\right), \theta^{*} \leq \theta \leq 1$. $w_{1}\left(\alpha_{i}, \beta_{i}, \gamma_{i}, \theta\right)=\frac{4 \theta^{3}(1-\theta)^{3}}{3 q_{i}(\theta) D_{1}^{2} D_{2}^{2}}[K-L]$,

$K=D_{1}^{2}\left(\left(\beta_{i}^{2}-\alpha_{i} \gamma_{i}\right)(1-\theta)^{2}+\left(\gamma_{i}^{2}+\beta_{i} \gamma_{i}\right)(1-\theta)\right)^{3 / 2}$,

$L=D_{2}^{2}\left(\theta \alpha_{i}\left(\alpha_{i}+\beta_{i}\right)+\left(\beta_{i}^{2}-\alpha_{i} \gamma_{i}\right) \theta^{2}\right)^{3 / 2}$,

$D_{1}=\alpha_{i}\left(1+\theta+\theta^{2}\right)+\beta_{i} \theta(1+2 \theta)+\gamma_{i} \theta^{2}$,

$D_{2}=\alpha_{i}(1-\theta)^{2}+\beta_{i}(1-\theta)(3-2 \theta)+\gamma_{i}\left(\theta^{2}-3 \theta+3\right)$,

$w_{2}\left(\alpha_{i}, \beta_{i}, \gamma_{i}, \theta\right)=-w_{1}\left(\alpha_{i}, \beta_{i}, \gamma_{i}, \theta\right), \theta^{*}=\frac{\alpha_{i}+\beta_{i}}{\alpha_{i}+2 \beta_{i}+\gamma_{i}}$.

Proof. Let $f(x)$ be the function to be interpolated by the $C^{2}$-rational quintic function (1). It is assumed that $f(x)$ is a $C^{3}$-continuous function, i.e., the order of continuity of $f(x)$ is greater than the order of continuity of its interpolating function $S(x)$. Absolute error $E[f]$ of interpolation by equation (1) is given by

$E[f]=|f(x)-S(x)| \leq\left|f^{(3)}(\tau)\right| \frac{1}{2} \int_{x_{i}}^{x_{i+1}}\left|E_{x}(g(\tau))\right| d \tau$.

Here, $\quad g(\tau)=(x-\tau)_{+}^{2}, \quad E_{x}[g(\tau)]=r_{1}(\tau, x), \quad$ for $\tau \in\left[x_{i}, x\right]$ and $E_{x}[g(\tau)]=r_{2}(\tau, x)$, for $\tau \in\left[x, x_{i+1}\right]$.

Using Duan et al. (2007), explicit value of $E_{x}(g(\tau))$ over the intervals $\left[x_{i}, x\right]$ and $\left[x, x_{i+1}\right]$ are the following:

$r_{1}(\tau, x)=(x-\tau)^{2}-\frac{\omega_{i}(\theta)}{q_{i}(\theta)}, r_{2}(\tau, x)=-\frac{\omega_{i}(\theta)}{q_{i}(\theta)}$,

$\omega_{i}(\theta)=\sum_{j=3}^{5}(1-\theta)^{5-j} \theta^{j} c_{j}$,

$c_{3}=\left(3 \gamma_{i_{i}}+3 \beta_{i}+\alpha_{i}\right)\left(x_{i+1}-\tau\right)^{2}-2 h_{i}\left(2 \gamma_{i}+\beta_{i}\right)\left(x_{i+1}-\tau\right)+\gamma_{i} h_{i}^{2}$,

$c_{4}=\left(3 \gamma_{i}+\beta_{i}\right)\left(x_{i+1}-\tau\right)^{2}-2 \gamma_{i} h_{i}\left(x_{i+1}-\tau\right), c_{5}=\gamma_{i}\left(x_{i+1}-\tau\right)^{2}$.

To evaluate the integral (9), firstly the integrals, $\quad \int_{x_{i}}^{x}\left|r_{1}(\tau, x)\right| d \tau \quad$ and $\int_{x}^{x_{i+1}}\left|r_{2}(\tau, x)\right| d \tau$ are evaluated. Following observations are made about the functions $r_{1}(\tau, x)$ and $r_{2}(\tau, x)$ : $r_{1}(\tau, x)$ is a quadratic polynomial in $\tau$. By the construction, $r_{1}(\tau, x)=0$ at $\tau=x_{i}$. Its value at $\tau=x$ after simplification is $r_{1}(x, x)=\frac{-h_{i}^{2}(1-\theta)^{3} \theta^{3}\left\{\alpha_{i}(1-\theta)+\beta_{i}(1-2 \theta)-\gamma_{i} \theta\right\}}{q_{i}(\theta)}$. Root of $r_{1}(x, x)$ is $\theta^{*}=\frac{\alpha_{i}+\beta_{i}}{\alpha_{i}+2 \beta_{i}+\gamma_{i}}$, and it lies in the interval $(0,1)$. By simple calculation it is noted that when $\theta \leq \theta^{*}, r_{1}(x, x) \leq 0$ and when $\theta \geq \theta^{*}, r_{1}(x, x) \geq 0$. Using quadratic formula, the following roots of $r_{1}(\tau, x)$ in $\left(x_{i}, x\right)$ are found: 
$\tau_{1}^{*}=x-\frac{M_{1}+N_{1}}{D_{1}}, \tau_{2}^{*}=x-\frac{M_{1}-N_{1}}{D_{1}}$,

$M_{1}=h_{i} \theta^{3}\left(\alpha_{i}+2 \beta_{i}+\gamma_{i}\right), N_{1}=h_{i} \theta \sqrt{\theta\left\{\alpha_{i}\left(\alpha_{i}+\beta_{i}\right)+\left(\beta_{i}^{2}-\alpha_{i} \gamma_{i}\right) \theta\right\}}$,

$D_{1}=\alpha_{i}\left(1+\theta+\theta^{2}\right)+\beta_{i} \theta(1+2 \theta)+\gamma_{i} \theta^{2}$.

If $\theta<\theta^{*}$ then $\tau_{1}^{*}<\tau_{2}^{*}$ and $r_{1}(x, x)<0$, so the integral of $r_{1}(\tau, x)$ for $\tau \in\left[x_{i}, x\right]$, is calculated as:

$$
\begin{aligned}
I_{1}= & \int_{x_{i}}^{x}\left|r_{1}(\tau, x)\right| d \tau \\
= & \int_{x_{i}}^{\tau_{1}^{*}}-\left(r_{1}(\tau, x)\right) d \tau+\int_{\tau_{1}^{*}}^{\tau_{2}^{*}} r_{1}(\tau, x) d \tau+\int_{\tau_{2}^{*}}^{x}-\left(r_{1}(\tau, x)\right) d \tau \\
= & \frac{-h_{i}^{3} \theta^{3}(1-\theta)^{3}}{3 q_{i}(\theta) D_{1}^{2}}\left[\left(\alpha_{i}\left(1+\theta+\theta^{2}\right) .\right.\right. \\
& \left.\quad+\beta_{i} \theta(1+2 \theta)+\gamma_{i} \theta^{2}\right)^{2}\left(\alpha_{i}(1-\theta)^{2}-2 \beta_{i} \theta(1-\theta)\right. \\
& \left.\left.\quad+\gamma_{i} \theta^{2}\right)+8\left(\theta\left(\left(\alpha_{i}+\beta_{i}\right)+\left(\beta_{i}^{2}-\alpha_{i} \gamma_{i}\right) \theta\right)\right)^{3 / 2}\right] \ldots(11)
\end{aligned}
$$

If $\theta>\theta^{*}$, then $r_{1}(x, x)>0$ and $\tau_{1}^{*}<\tau_{2}^{*}$. Then value of the integral is calculated as follows:

$$
\begin{aligned}
\int_{x_{i}}^{x}\left|r_{1}(\tau, x)\right| d \tau & =\int_{x_{i}}^{\tau_{1}^{*}} r_{1}(\tau, x) d \tau+\int_{\tau_{1}^{*}}^{\tau_{2}^{*}}-\left(r_{1}(\tau, x)\right) d \tau \\
& +\int_{\tau_{2}^{*}}^{x} r_{1}(\tau, x) d \tau=-I_{1}
\end{aligned}
$$

Now, consider $r_{2}(\tau, x), \tau \in\left[x, x_{i+1}\right]$ given in equation (10). $r_{2}(\tau, x)$ is also quadratic in $\tau$. It is also observed that $r_{2}\left(x_{i+1}, x\right)=0$, and $r_{2}(x, x)=r_{1}(x, x)$. Since $r_{2}(\tau, x)$ defined in (10) is quadratic in $\tau$, roots of $r_{2}(\tau, x)$ by quadratic formula are:

$$
\begin{aligned}
& \tau_{*}^{1}=x_{i+1}-\frac{M_{2}+N_{2}}{D_{2}}, \tau_{*}^{2}=x_{i+1}-\frac{M_{2}-N_{2}}{D_{2}}, \\
& M_{2}=h_{i}(1-\theta)\left(\beta_{i}(1-\theta)+\gamma_{i}(2-\theta)\right), \\
& N_{2}=h_{i}(1-\theta) \sqrt{\beta_{i}^{2}(1-\theta)^{2}+\gamma_{i}^{2}(1-\theta)+\beta_{i} \gamma_{i}(1-\theta)-\alpha_{i} \gamma_{i}(1-\theta)^{2}}, \\
& D_{2}=\alpha_{i}(1-\theta)^{2}+\beta_{i}(1-\theta)(3-2 \theta)+\gamma_{i}\left(\theta^{2}-3 \theta+3\right) .
\end{aligned}
$$

Both of these roots lie in the interval $\left[x, x_{i+1}\right]$. By comparing $\tau_{*}^{1}$ and $\tau_{*}^{2}$, it is observed that $\tau_{*}^{1}<\tau_{*}^{2}$.
Since for $\theta<\theta^{*}, r_{2}(x, x)$ is negative so the integral of absolute value of $r_{2}(\tau, x)$ is calculated as follows:

$$
\begin{aligned}
I_{2}= & \int_{x}^{x_{i+1}}\left|r_{2}(\tau, x)\right| d \tau \\
= & \int_{x}^{\tau_{*}^{1}}-\left(r_{2}(\tau, x)\right) d \tau+\int_{\tau_{*}^{1}}^{\tau_{*}^{2}} r_{2}(\tau, x) d \tau+\int_{\tau_{*}^{2}}^{x_{i+1}}-\left(r_{2}(\tau, x)\right) d \tau \\
= & \frac{h_{i}^{3} \theta^{3}(1-\theta)^{3}}{3 q_{i}(\theta) D_{2}^{2}}\left[\left(\alpha_{i}(1-\theta)^{2}+\beta_{i}(1-\theta)(3-2 \theta)+\gamma_{i}\left(\theta^{2}-3 \theta+3\right)\right)^{2} \times\right. \\
& \quad\left(\alpha_{i}(1-\theta)^{2}-2 \beta_{i} \theta(1-\theta)+\gamma_{i} \theta^{2}\right)+8\left(\left(\beta_{i}^{2}-\alpha_{i} \gamma_{i}\right)(1-\theta)^{2}\right. \\
& \left.+\left(\gamma_{i}^{2}+\beta_{i} \gamma_{i}\right)(1-\theta)\right]
\end{aligned}
$$

If $\theta>\theta^{*}, r_{2}(x, x)>0$, so the value of integral of $\left|r_{2}(\tau, x)\right|$

$$
\begin{aligned}
\int_{x}^{x_{i+1}}\left|r_{2}(\tau, x)\right| d \tau & =\int_{x}^{\tau_{*}^{1}} r_{2}(\tau, x) d \tau+\int_{\tau_{*}^{1}}^{\tau_{*}^{2}}-\left(r_{2}(\tau, x)\right) d \tau \\
& +\int_{\tau_{*}^{2}}^{x_{i+1}} r_{2}(\tau, x) d \tau=-I_{2}
\end{aligned}
$$

Thus, combining equations (11) and (13), the computed absolute error of interpolation for $\theta \leq \theta^{*}$ is given in equation (15).

$|f(x)-S(x)| \leq \frac{\left\|f^{(3)}\right\|}{2} \int_{x_{i}}^{x_{i+1}} R_{x}\left[(x-\tau)_{+}^{2}\right] d \tau=\left\|f^{(3)}\right\| h_{i}^{3} w_{1}\left(\alpha_{i}, \beta_{i}, \gamma_{i}, \theta\right)$.

Here, $w_{1}\left(\alpha_{i}, \beta_{i}, \gamma_{i}, \theta\right)=\frac{4 \theta^{3}(1-\theta)^{3}}{3 q_{i}(\theta) D_{1}^{2} D_{2}^{2}}[K-L]$,

$K=D_{1}^{2}\left(\left(\beta_{i}^{2}-\alpha_{i} \gamma_{i}\right)(1-\theta)^{2}+\left(\gamma_{i}^{2}+\beta_{i} \gamma_{i}\right)(1-\theta)\right)^{3 / 2}$,

$L=D_{2}^{2}\left(\theta\left\{\alpha_{i}\left(\alpha_{i}+\beta_{i}\right)+\left(\beta_{i}^{2}-\alpha_{i} \gamma_{i}\right) \theta\right\}\right)^{3 / 2}$.

If $\theta \geq \theta^{*}$, combining equations (12) and (14), we have

$|f(x)-S(x)| \leq \frac{\left\|f^{(3)}\right\|}{2} \int_{x_{i}}^{x_{i+1}} R_{x}\left[(x-\tau)_{+}^{2}\right] d \tau=\left\|f^{(3)}\right\| h_{i}^{3} w_{2}\left(\alpha_{i}, \beta_{i}, \gamma_{i}, \theta\right)$.

Here, $w_{2}\left(\alpha_{i}, \beta_{i}, \gamma_{i}, \theta\right)=-w_{1}\left(\alpha_{i}, \beta_{i}, \gamma_{i}, \theta\right)$. The expressions (9) and (10) complete the proof.

Remark 1. In the preceding theorem it is assumed that $f(x)$ is a $C^{3}$-continuous function. Error of approximation can be calculated in the same way while interpolating the $C^{k}$-continuous function $f(x)$ using $C^{2}$-rational quintic function (1). Using Duan et al. (2007), the maximum value of optimal error coefficient $c_{i}$ defined in Theorem 1 is calculated which is $c_{i}=0.0219$. 


\section{Positivity-preserving $C^{2}$-rational quintic function}

Given 2D positive data $\left\{\left(x_{i}, f_{i}\right): i=0,1,2, \ldots, n\right\}$ defined over the interval $[a, b]$, a $C^{2}$-rational quintic scheme is introduced to interpolate the $2 \mathrm{D}$ positive data $\left(f_{i}>0\right)$ as positive curve. Because the first order derivatives $\left(d_{i}\right)$ as well as second order derivatives $\left(D_{i}\right)$ at the knots $x_{i}, i=0,1,2, \ldots, n$, are provided, range of the free parameters $\left(\alpha_{i}, \beta_{i}, \gamma_{i}\right)$ is calculated to construct a positive $C^{2}$-rational quintic function (1) on $[a, b]$.

The $C^{2}$-rational quintic function $S(x)$ defined in equation (1) is positive if $p_{i}(\theta)>0$ and $q_{i}(\theta)>0$.

As $0 \leq \theta \leq 1$, and free parameters $\left(\alpha_{i}, \beta_{i}, \gamma_{i}\right)$, are positive real numbers $q_{i}(\theta)$ is always positive. Now the problem of positivity of $S(x)$ is reduced to the problem of positivity of $p_{i}(\theta)$. The quintic polynomial $p_{i}(\theta)>0$ if each of $A_{i}>0$ is positive. It is clear that $A_{0}$ and $A_{5}$ are always positive by the given hypothesis. Now, $p_{i}(\theta)$ is positive if $A_{i}>0, i=1,2,3,4$.

$$
\begin{aligned}
& A_{1}>0 \text { if } \beta_{i}>\frac{-\alpha_{i} h_{i} d_{i}}{f_{i}} . \\
& \text { For } 3 f_{i}+h_{i} d_{i}>0, A_{2}>0 \text { if } \beta_{i}>\frac{-\alpha_{i} h_{i}\left(4 d_{i}+h_{i} D_{i}\right)}{2\left(3 f_{i}+h_{i} d_{i}\right)} . \\
& \text { For } 3 f_{i}+h_{i} d_{i}<0, A_{2}>0 \text { if } \beta_{i}<\frac{-\alpha_{i} h_{i}\left(4 d_{i}+h_{i} D_{i}\right)}{2\left(3 f_{i}+h_{i} d_{i}\right)} .
\end{aligned}
$$$$
\text { For } 3 f_{i+1}-h_{i} d_{i+1}>0, A_{3}>0 \text { if } \beta_{i}>\frac{\gamma_{i} h_{i}\left(4 d_{i+1}-h_{i} D_{i+1}\right)}{2\left(3 f_{i+1}-h_{i} d_{i+1}\right)} \text {. }
$$$$
\text { For } 3 f_{i+1}-h_{i} d_{i+1}<0, A_{3}>0 \text { if } \beta_{i}<\frac{\gamma_{i} h_{i}\left(4 d_{i+1}-h_{i} D_{i+1}\right)}{2\left(3 f_{i+1}-h_{i} d_{i+1}\right)} \text {. }
$$$$
A_{4}>0 \text { if } \beta_{i}>\frac{\gamma_{i} h_{i} d_{i+1}}{f_{i+1}} .
$$

The above discussion leads to four cases depending upon the values of $3 f_{i}+h_{i} d_{i}$ and $3 f_{i+1}-h_{i} d_{i+1}$ in each subinterval. These cases can be avoided by replacing $h_{i}$ with $h_{i} / k_{i}$ in $A_{i}, i=0,1,2,3,4$, where $k_{i}$ is introduced as a positive real number. Hence the constraints on free parameters to assure positivity of $A_{i}, i=0,1,2,3,4$, are calculated as follows:

$$
\begin{aligned}
& A_{1}>0 \text { if } \beta_{i}>\frac{-\alpha_{i} \widetilde{h}_{i} d_{i}}{f_{i}} . \\
& A_{2}>0 \text { if } \beta_{i}>\frac{-\alpha_{i} \widetilde{h}_{i}\left(4 d_{i}+\widetilde{h}_{i} D_{i}\right)}{2\left(3 f_{i}+\widetilde{h}_{i} d_{i}\right)} \text { and } k_{i}>\frac{-h_{i} d_{i}}{3 f_{i}} . \\
& A_{3}>0 \text { if } \beta_{i}>\frac{\gamma_{i} \widetilde{h}_{i}\left(4 d_{i+1}-\widetilde{h}_{i} D_{i+1}\right)}{2\left(3 f_{i+1}-\widetilde{h}_{i} d_{i+1}\right)} \text { and } k_{i}>\frac{h_{i} d_{i+1}}{3 f_{i+1}} . \\
& A_{4}>0 \text { if } \beta_{i}>\frac{\gamma_{i} \widetilde{h}_{i} d_{i+1}}{f_{i+1}}, \widetilde{h}_{i}=\frac{h_{i}}{k_{i}}
\end{aligned}
$$

All of the above discussion is summarised in the following theorem.

Theorem 2. The $C^{2}$-rational quintic function (1) preserves the positive shape of $2 \mathrm{D}$ data if in each subinterval $\left[x_{i}, x_{i+1}\right]$ shape parameters $\alpha_{i}, \beta_{i}$ and $\gamma_{i}$ satisfy the following constraints:

$\alpha_{i}>0, \gamma_{i}>0$,

$\beta_{i}>\max \left\{0, \delta_{1}, \delta_{2}, \delta_{3}, \delta_{4}\right\}$ and $k_{i}>\max \left\{0, \delta_{5}, \delta_{6}\right\}$,

where

$\delta_{1}=\frac{-\alpha_{i} \tilde{h}_{i} d_{i}}{f_{i}}, \delta_{2}=\frac{-\alpha_{i} \tilde{h}_{i}\left(4 d_{i}+\tilde{h}_{i} D_{i}\right)}{2\left(3 f_{i}+\tilde{h}_{i} d_{i}\right)}, \delta_{3}=\frac{\gamma_{i} \tilde{h}_{i}\left(4 d_{i+1}-\tilde{h}_{i} D_{i+1}\right)}{2\left(3 f_{i+1}-\tilde{h}_{i} d_{i+1}\right)}$,

$\delta_{4}=\frac{\gamma_{i} \tilde{h}_{i} d_{i+1}}{f_{i+1}}, \quad \delta_{5}=\frac{-h_{i} d_{i}}{3 f_{i}}, \quad \delta_{6}=\frac{h_{i} d_{i+1}}{3 f_{i+1}}, \tilde{h}_{i}=\frac{h_{i}}{k_{i}}$.

These constraints can also be rewritten as:

$\beta_{i}=u_{i}+\max \left\{0, \delta_{1}, \delta_{2}, \delta_{3}, \delta_{4}\right\}, k_{i}=v_{i}+\max \left\{0, \delta_{5}, \delta_{6}\right\}$,

where $u_{i}$ and $v_{i}$ are real positive constants.

\section{Algorithm 1.}

Step 1. Input the 2D positive data set $\left\{\left(x_{i}, f_{i}\right): i=0,1,2, \ldots, n\right\}$.

Step 2. Compute the values of first order derivatives $d_{i}$ at knots $x_{i}, i=0,1,2, \ldots, n$ by the arithmetic mean approximation scheme of derivatives given in equation (3).

Step 3. Compute the values of second order derivatives $D_{i}$, at knots $\mathrm{x}_{\mathrm{i}}, \mathrm{i}=0,1,2, \ldots, \mathrm{n}$ by the arithmetic mean approximation scheme of derivatives given in equation (4).

(If the first and second order derivatives at knots are given as input with $2 \mathrm{D}$ positive data then skip the Steps 2 and 3, and go to Step 4).

Step 4. Use the data accumulated in Steps 1, 2 and 3 to calculate the values of shape parameters using Theorem 2.

Step 5. Substitute these values of $f_{i}, d_{i}, D_{i}$ and shape parameters in $C^{2}$-rational quintic function (1) to obtain the positive curve for given $2 \mathrm{D}$ positive data.

\section{Range restricted $C^{2}$-rational quintic function}

In this section, an interpolating scheme is introduced to preserve the shape of $2 \mathrm{D}$ data lying above the line using rational quintic function (1). 
Let $\left\{a=x_{0}, x_{1}, x_{2}, \ldots, x_{n}=b\right\}$ be the partition of the interval $[a, b]$. Let $f_{i}$ be ordinate values at $x_{i}$, $i=0,1,2, \ldots, n$, and lying above the straight line $y=m x+c$. Here $m$ is the slope of given line and $y$-intercept is $c$. This hypothesis is expressed mathematically in equation (17).

$f_{i}>m x_{i}+c$.

The rational quintic function (1) interpolating the given dataset $\left\{\left(x_{i}, f_{i}\right), i=0,1, \ldots n\right\}$ will lie above the straight line $y=m x+c$ if $S(x)>m x+c, \forall x \in[a, b]$.

In each subinterval $\left[x_{i}, x_{i+1}\right]$ by expressing the straight line in terms of parameter $\theta$, the above equation takes the following form:

$S(x)>a_{i}(1-\theta)+b_{i} \theta$

Here, $a_{i}=m x_{i}+c, b_{i}=m x_{i+1}+c$ and $b_{i}=m h_{i}$ $+a_{i}, h_{i}=x_{i+1}-x_{i}$.

Substituting the value $S(x)=\frac{p_{i}(\theta)}{q_{i}(\theta)}$, in equation (18), we have $\frac{p_{i}(\theta)}{q_{i}(\theta)}>a_{i}(1-\theta)+b_{i} \theta$.

After some simplifications, we have

$V_{i}(\theta)=\sum_{j=0}^{5}(1-\theta)^{5-j} \theta^{j} B_{j}>0$,

where

$$
\begin{aligned}
B_{0} & =\alpha_{i}\left(f_{i}-a_{i}\right), \\
B_{1} & =\left(3 \alpha_{i}+\beta_{i}\right) f_{i}+\alpha_{i} h_{i} d_{i}-\left(2 \alpha_{i}+\beta_{i}\right) a_{i}-\alpha_{i} b_{i}, \\
B_{2} & =\left(3 \alpha_{i}+3 \beta_{i}+\gamma_{i}\right) f_{i}+\left(2 \alpha_{i}+\beta_{i}\right) h_{i} d_{i}+0.5\left(\alpha_{i} h_{i}^{2} D_{i}\right) \\
& -\left(2 \beta_{i}+\alpha_{i}+\gamma_{i}\right) a_{i}-\left(2 \alpha_{i}+\beta_{i}\right) b_{i}, \\
B_{3}= & \left(3 \gamma_{i}+3 \beta_{i}+\alpha_{i}\right) f_{i+1}-\left(2 \gamma_{i}+\beta_{i}\right) h_{i} d_{i+1}+0.5\left(\gamma_{i} h_{i}^{2} D_{i+1}\right) \\
& -\left(2 \beta_{i}+\alpha_{i}+\gamma_{i}\right) b_{i}-\left(2 \gamma_{i}+\beta_{i}\right) a_{i}, \\
B_{4} & =\left(3 \gamma_{i}+\beta_{i}\right) f_{i+1}-\gamma_{i} h_{i} d_{i+1}-\left(2 \gamma_{i}+\beta_{i}\right) b_{i}-\gamma_{i} a_{i}, \\
B_{5} & =\gamma_{i}\left(f_{i+1}-b_{i}\right) .
\end{aligned}
$$

Thus $V_{i}(\theta)>0$ if $B_{j}>0, j=0,1,2,3,4,5$. Because of the hypothesis (17), $B_{0}$ and $B_{5}$ are positive. After replacing $h_{i}$ by $h_{i} / k_{i}$ in $B_{1}, B_{2}, B_{3}$ and $B_{4}$ the constraints on free parameter are calculated as follows to ensure $V_{i}(\theta)>0$ over the whole domain.
Now,

$B_{1}>0$ if $\beta_{i}>\frac{-\alpha_{i}\left(f_{i}+\widetilde{h}_{i} d_{i}-b_{i}\right)}{\left(f_{i}-a_{i}\right)}$, where $\tilde{h}_{i}=\frac{h_{i}}{k_{i}}, k_{i}>0$.

$B_{2}>0$ if $\beta_{i}>\frac{\left.-\alpha_{i} 4\left(f_{i}+\widetilde{h}_{i} d_{i}-b_{i}\right)+\tilde{h}_{i}^{2} D_{i}\right\}}{2\left(3 f_{i}+\tilde{h}_{i} d_{i}-2 a_{i}-b_{i}\right)}$, and $k_{i}>\frac{h_{i}\left(m-d_{i}\right)}{3\left(f_{i}-a_{i}\right)}$.

$B_{3}>0$ if $\beta_{i}>\frac{-\gamma_{i}\left\{4\left(f_{i+1}-\widetilde{h}_{i} d_{i+1}-a_{i}\right)+\widetilde{h}_{i}^{2} D_{i+1}\right\}}{2\left(3 f_{i+1}-\widetilde{h}_{i} d_{i+1}-2 b_{i}-a_{i}\right)}$, and $k_{i}>\frac{h_{i}\left(d_{i+1}-m\right)}{3\left(f_{i+1}-b_{i}\right)}$.

$B_{4}>0$ if $\beta_{i}>\frac{-\gamma_{i}\left(f_{i+1}-\widetilde{h}_{i} d_{i+1}-a_{i}\right)}{\left(f_{i+1}-b_{i}\right)}$.

Here, $k_{i}$ is introduced as positive real free parameter. The above discussion is summarised in Theorem 3 .

Theorem 3. The $C^{2}$-rational quintic function (1) preserves the shape of data lying above a straight line $y=m x+c$ if the shape parameters $\alpha_{i}, \beta_{i}$ and $\gamma_{i}$ satisfy the following constraints:

$\alpha_{i}>0, \gamma_{i}>0$,

$\beta_{i}>\max \left\{0, \rho_{1}, \rho_{2}, \rho_{3}, \rho_{4}\right\}$ and $k_{i}>\max \left\{0, \rho_{5}, \rho_{6}\right\}$,

$\rho_{1}=\frac{-\alpha_{i}\left(f_{i}+\widetilde{h}_{i} d_{i}-b_{i}\right)}{\left(f_{i}-a_{i}\right)}, \rho_{2}=\frac{-\alpha_{i}\left\{4\left(f_{i}+\widetilde{h}_{i} d_{i}-b_{i}\right)+\widetilde{h}^{2} D_{i}\right\}}{2\left(3 f_{i}+\widetilde{h}_{i} d_{i}-2 a_{i}-b_{i}\right)}$,

$\rho_{3}=\frac{-\gamma_{i}\left(f_{i+1}-\widetilde{h}_{i} d_{i+1}-a_{i}\right)}{\left(f_{i+1}-b_{i}\right)}, \rho_{4}=\frac{-\gamma_{i}\left\{4\left(f_{i+1}-\widetilde{h}_{i} d_{i+1}-a_{i}\right)+\widetilde{h}_{i}^{2} D_{i+1}\right\}}{2\left(3 f_{i+1}-\widetilde{h}_{i} d_{i+1}-2 b_{i}-a_{i}\right)}$,

, $\rho_{5}=\frac{h_{i}\left(m-d_{i}\right)}{3\left(f_{i}-a_{i}\right)}, \rho_{6}=\frac{h_{i}\left(d_{i+1}-m\right)}{3\left(f_{i+1}-b_{i}\right)}$.

These constraints can also be rewritten as:

$\beta_{i}=\hat{u}_{i}+\max \left\{0, \rho_{1}, \rho_{2}, \rho_{3}, \rho_{4}\right\}$ and $k_{i}=\hat{v}_{i}+\max \left\{0, \rho_{5}, \rho_{6}\right\}$.

Here $\hat{u}_{i}$ and $\hat{v}_{i}$ are introduced as real positive free parameters.

\section{Algorithm 2.}

Step 1. Input the 2D data set $\left\{\left(x_{i}, f_{i}\right): i=0,1,2, \ldots, n\right\}$ lying above the line $y=m x+c$.

Step 2. Compute the values of $h_{i}=x_{i+1}-x_{i} ; a_{i}=$ $=m x_{i}+c$ and $b_{i}=m h_{i}+a_{i}$.

Step 3. Compute the values of first and second order derivatives $d_{i}$ and $D_{i}$ at knots $\mathrm{x}_{\mathrm{i}}, \mathrm{i}=0,1,2, \ldots, \mathrm{n}$ by the arithmetic mean approximation scheme of derivatives given in (3) and (4).

(If the first and second order derivatives at knots are given as input with 2D then skip the Step 3 and go to Step 4).

Step 4. Calculate the value of shape parameters using Theorem 3.

Step 5. Substitute the values of $f_{i}, d_{i}, D_{i}$ and shape parameters in $C^{2}$-rational quintic function (1) to obtain the curve lying above the straight line $y=m x+c$. 
Remark 2. It follows from the above discussion that if the given data consists of only functional values then the first and second order derivative values at knots will be computed by the arithmetic mean approximation scheme of derivatives. These values will be used further in Theorem 2 and Theorem 3. If the first and second order derivative values at knots are also prescribed with functional values then these values will be directly used in $C^{2}$-rational quintic function to get the required results.

\section{RESULTS AND DISCUSSION}

In the following numerical examples, the efficiency of the proposed shape-preserving schemes is demonstrated through graphical results. It is observed that the $C^{2}$-continuous quintic Hermite polynomial (Farin, 2005) fails to preserve the inherit shape of the datasets. However the $C^{2}$-continuous shape-preserving rational quintic function fills this gap in a pleasant way.

Example 1. Rivers are the main source of drinking water in most of the land areas of Pakistan, sepecifically in north Punjab. River water has less salt and it is more clear than sea water. Unfortunately different industrial (chemical and thermal waste), municipal (detergents, organic wastes) and agricultural (pesticides, fertilisers) material pollute the rivers. The pollutants enter to the rivers through different drains. In Hussain (2006), pollution load of five drains before entering the Chenab river was observed against the water load of these drains. It is a mixture of copper, lead, nickel, arsenic, sodium, nitrate, phosphate, etc. In Table 2, the quantity of lead in the pollution load of these five drains is provided along with their total wastage contribution in a day to the Chenab river (Hussain, 2006).

In Table 2, $n$ is drain number, $x$ is the waste contribution by drains in gallons and $y$ is the quantity of lead in milligrams (mg). The quantity of lead in waste contribution cannot be negative. The positive data in Table 2 is interpolated by two $C^{2}$-continuous functions, the quintic Hermite polynomial (Farin, 2005) and the $C^{2}$-continuous rational quintic function (1). Interpolation

Table 2: Lead contamination drains of Chenab river

\begin{tabular}{cccccc}
\hline$n$ & 1 & 2 & 3 & 4 & 5 \\
$x$ & 20 & 42 & 45 & 47 & 52 \\
$y$ & 0.05 & 0.06 & 1.5 & 1.65 & 1.4 \\
\hline
\end{tabular}

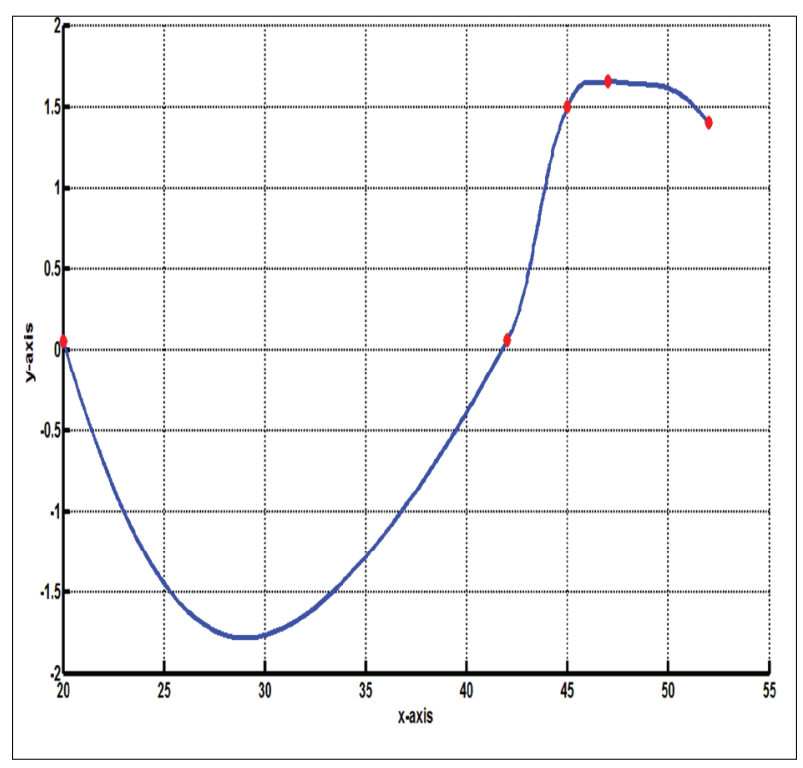

Figure 3: Quintic Hermite interpolation of dataset of Table 2

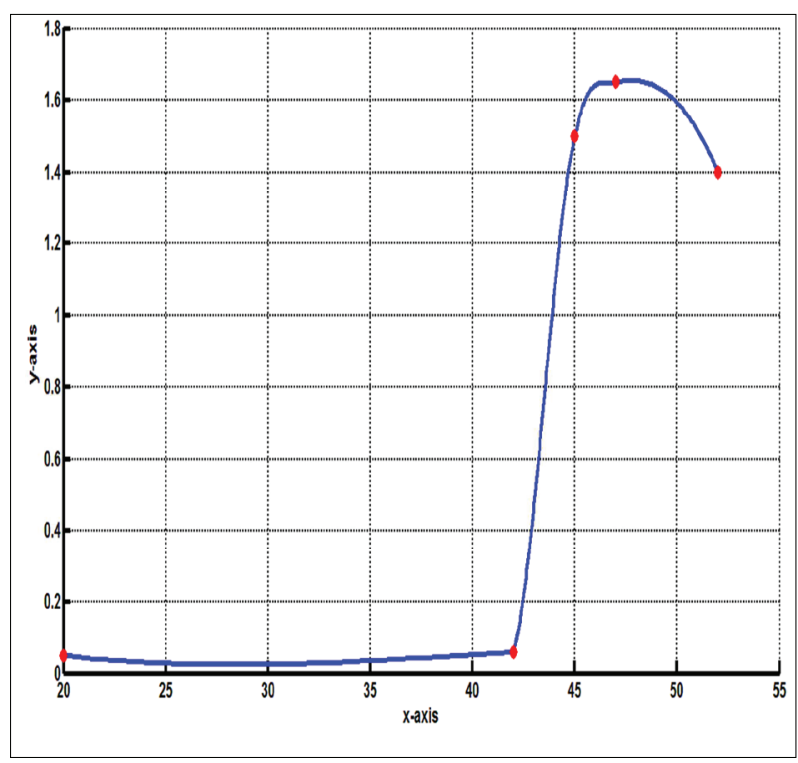

Figure 4: $\quad C^{2}$ - rational quintic function $\left(\alpha_{i}=0.005, \gamma_{i}=0.005, \forall i\right)$

of the data of Table 2 by the quintic Hermite polynomial is shown in Figure 3. It is clear from Figure 3 that the quintic Hermite loses the positivity. Interpolation of the same data by the $C^{2}$-positivity-preserving scheme of Theorem 2 is given in Figures 4 and 5. It is clear from Figures 4 and 5 that the interpolated curve is positive over the whole domain. Hence the positivity-preserving $C^{2}$-rational quintic interpolation scheme works efficiently. 


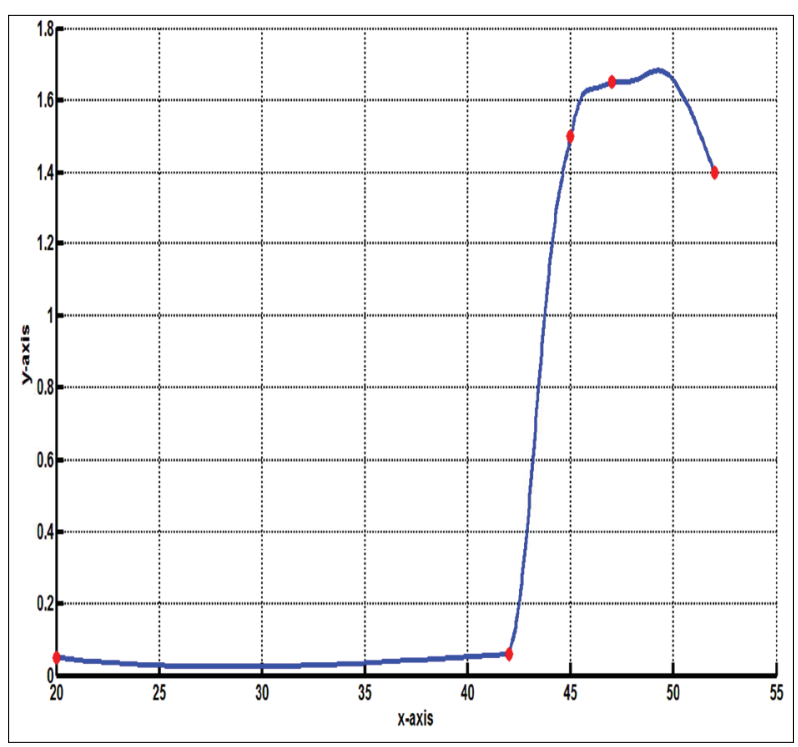

Figure 5: $\quad C^{2}$ - rational quintic function $\left(\alpha_{i}=1, \gamma_{i}=3, \forall i\right)$

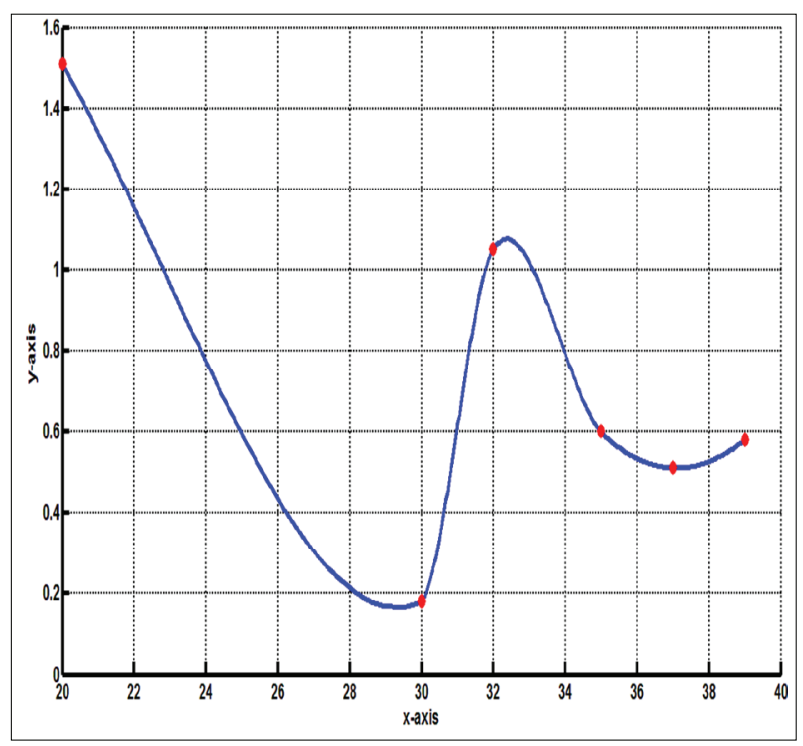

Figure 7: $\quad C^{2}$ - rational quintic function $\left(\alpha_{i}=0.004, \gamma_{i}=0.004, \forall i\right)$

Table 3: Creatinine level in human blood

\begin{tabular}{ccccccc}
\hline$x$ & 20 & 30 & 32 & 35 & 37 & 39 \\
$y$ & 1.51 & 0.18 & 1.05 & 0.6 & 0.51 & 0.58 \\
\hline
\end{tabular}

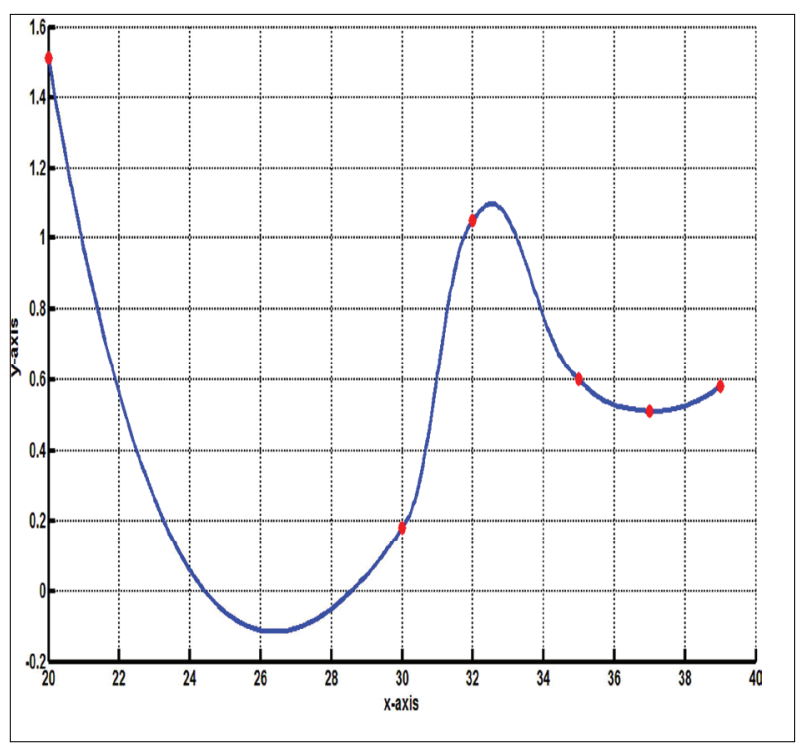

Figure 6: Quintic Hermite interpolation of dataset of Table 3

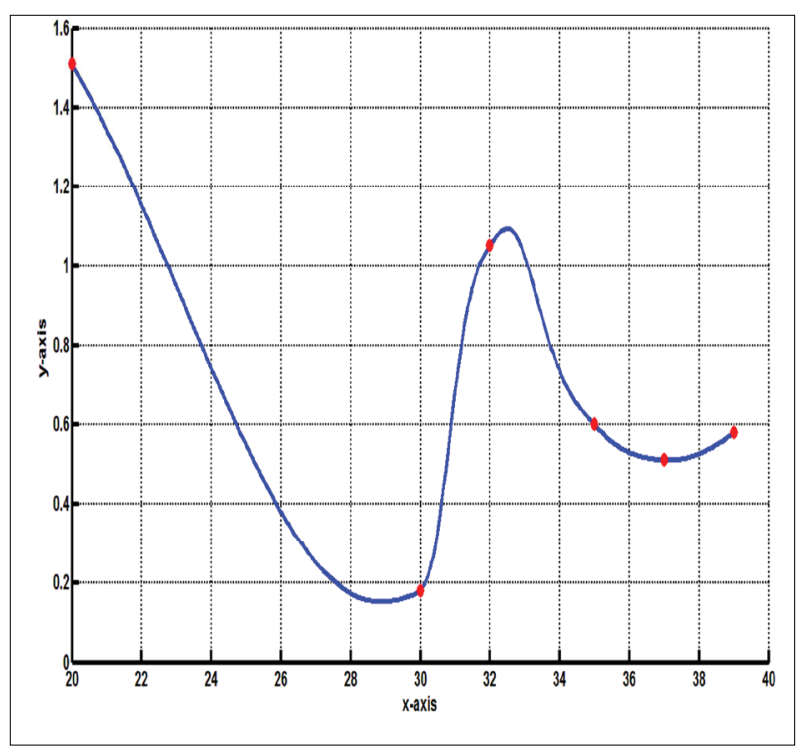

Figure 8: $\quad C^{2}$ - rational quintic function $\left(\alpha_{i}=2, \gamma_{i}=5, \forall i\right)$

Table 4: Data lying above the line $y=x+2$

\begin{tabular}{cccccc}
\hline$x$ & 0 & 4 & 21 & 30 & 32 \\
$y$ & 22.8 & 8 & 33.9 & 38.9 & 43.6 \\
\hline
\end{tabular}


Example 2. The amount of blood creatinine $\left(\mathrm{mgdL}^{-1}\right)$ is measured in six young individuals of age below forty. The observation of creatinine levels $(y)$ is compiled against the age $(x)$ of these individuals in Table 3. The normal range of creatinine is $0.50-0.90 \mathrm{mgdL}^{-1}$. It is clear from Table 3 that a 20 year individual is highly prompt to kidney desease. The second individual of age 30 years has a low creatinine level, which needs medical

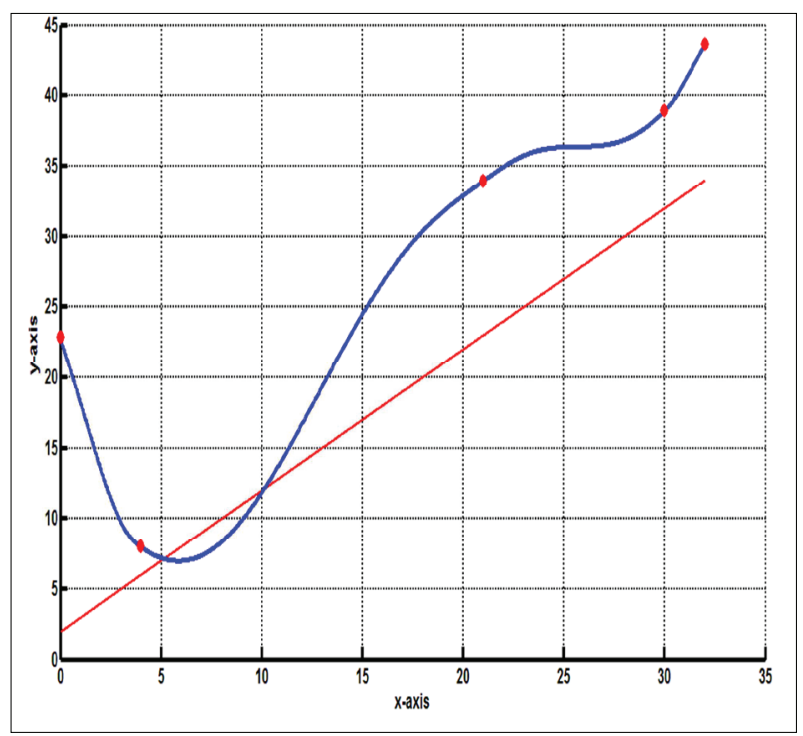

Figure 9: Quintic Hermite interpolation of dataset of Table 4

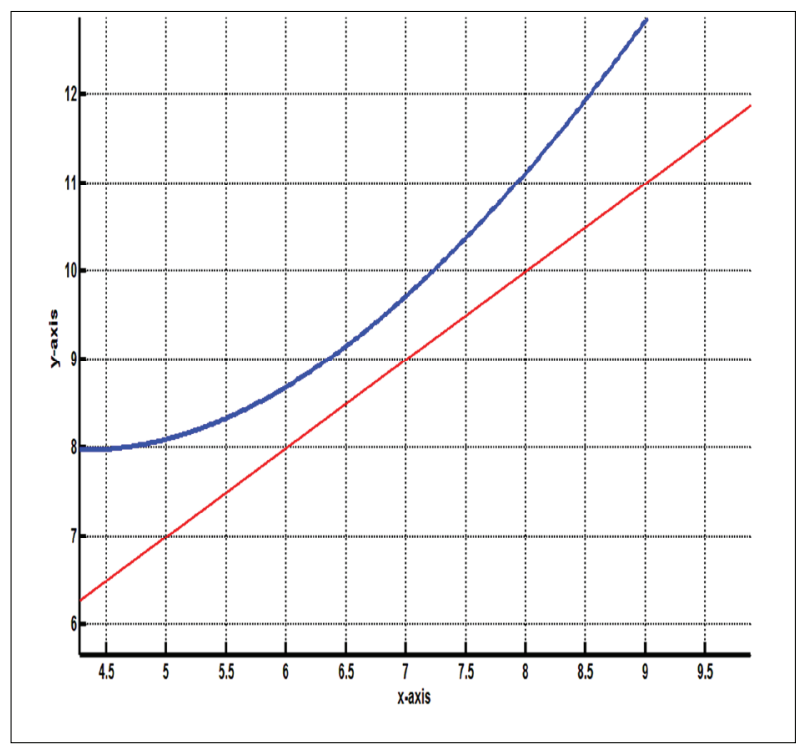

Figure 11: Close view of Figure 6 attention. Creatinine levels of all other individuals are acceptable.

Table 5: Data lying above the line $y=0.5 x-1$

\begin{tabular}{cccc}
\hline$x$ & 1 & 5.5 & 6 \\
$y$ & 1 & 2 & 6 \\
\hline
\end{tabular}

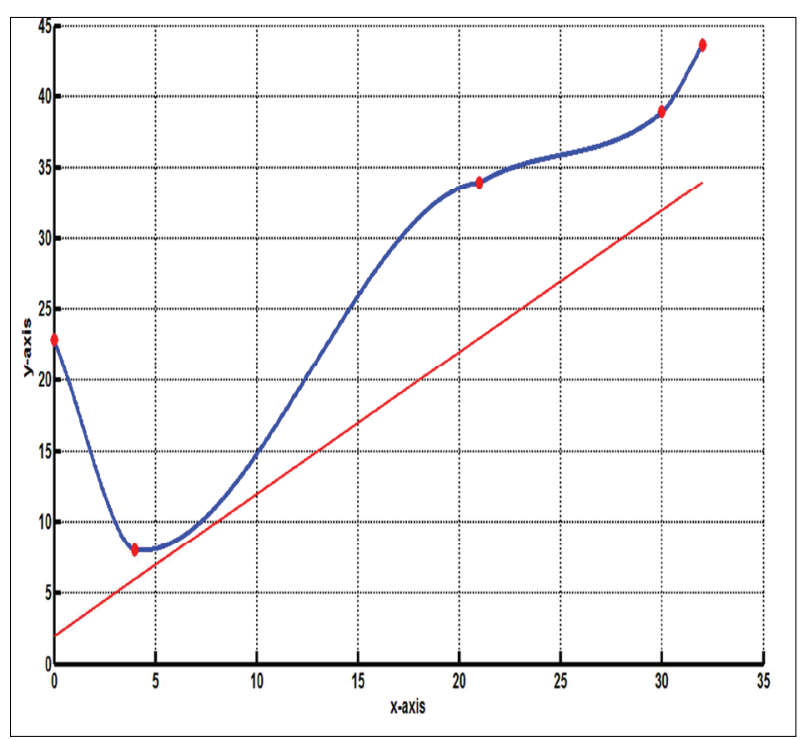

Figure 10: $C^{2}$-rational quintic function $\left(\alpha_{i}=0.0007, \gamma_{i}=0.0007, \forall i\right)$

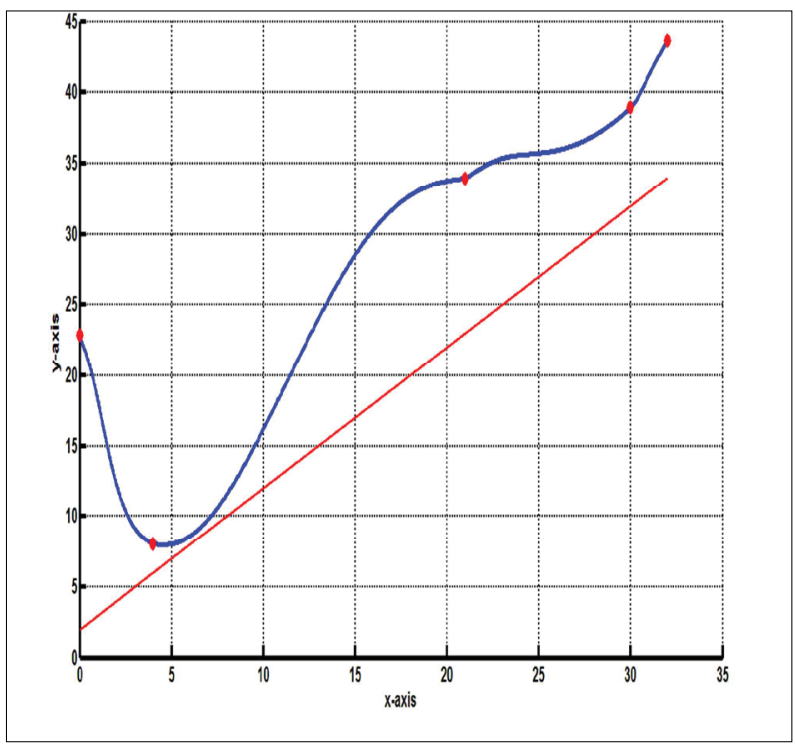

Figure 12: $C^{2}$ - rational quintic function $\left(\alpha_{i}=2, \gamma_{i}=10, \forall i\right)$ 


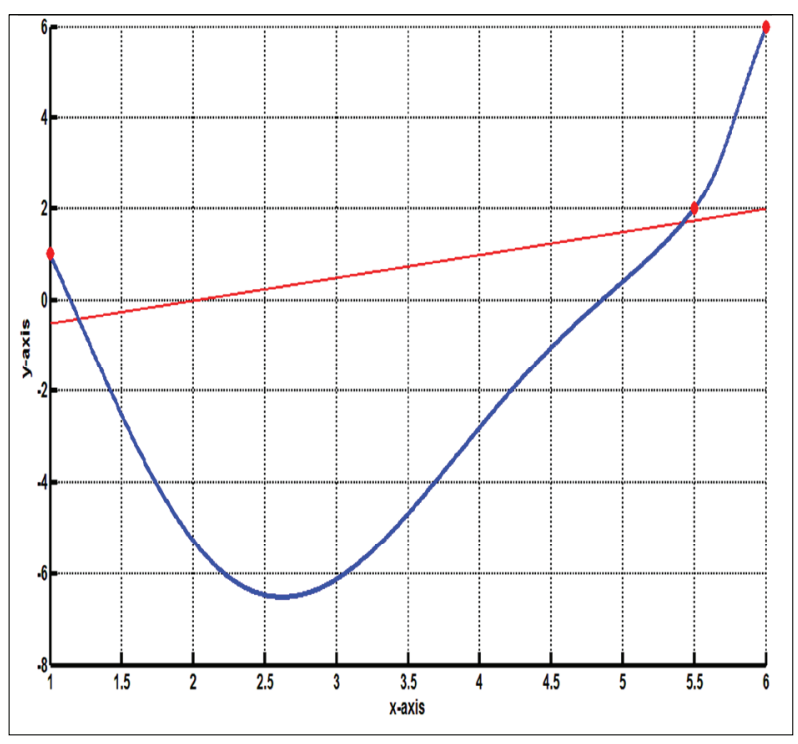

Figure 13: Quintic Hermite interpolation of dataset of Table 5

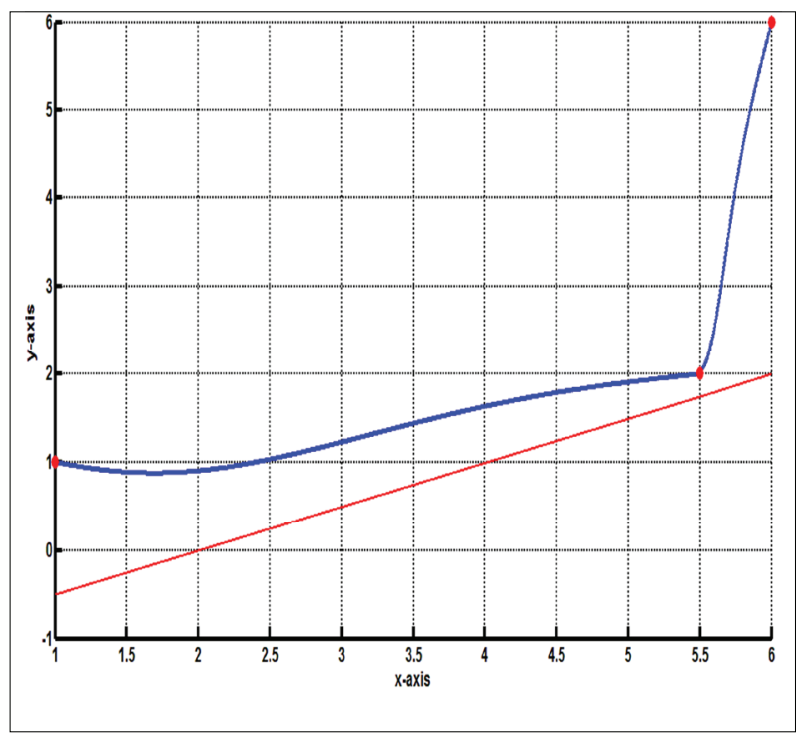

Figure 15: $C^{2}$-rational quintic function $\left(\alpha_{i}=2, \gamma_{i}=10, \forall i\right)$

The quintic Hermite interpolation of positive data of Table 3 is given in Figure 6. It is clear from Figure 6 that the positivity of data is lost in Figure 6 and it indicates that the people between age 25 to 30 have negative creatinine levels, which is not possible. In Figures 7 and 8 , the same data is interpolated by the $C^{2}$-positivitypreserving scheme of Theorem 2 . The interpolated curve in Figures 7 and 8 is positive everywhere. Hence the shape of positive data is preserved by rational quintic interpolant (1).

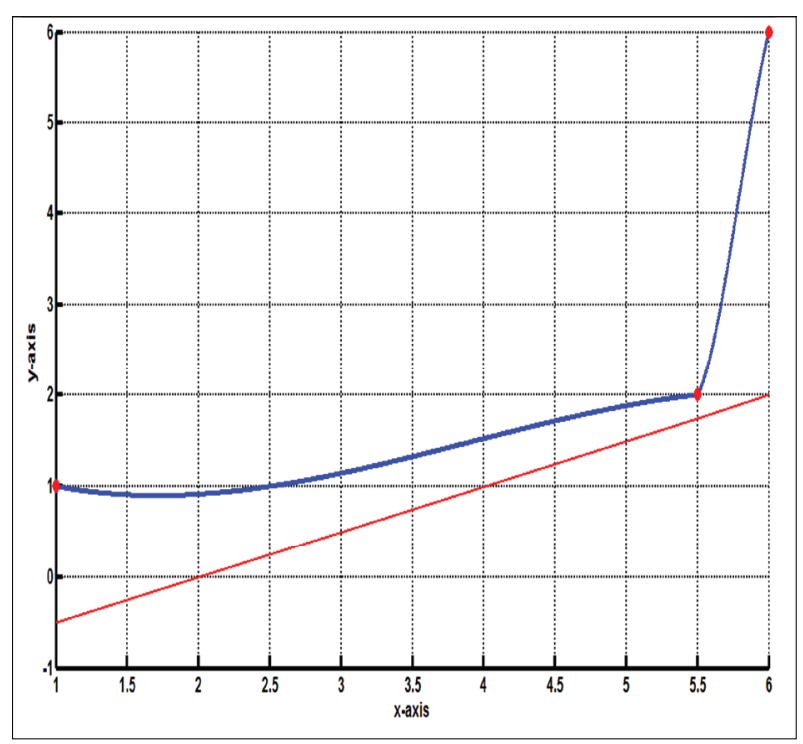

Figure 14: $C^{2}$ - rational quintic function $\left(\alpha_{i}=0.0300, \gamma_{i}=0.0300, \forall i\right)$

Example 3. The dataset in Table 4 is lying above the line $y=x+2$. In Figure 9, it is interpolated by $C^{2}$-quintic Hermite polynomial. Curve is lying below the line $y=x+2$ in Figure 9, i.e. the $C^{2}$ - quintic Hermite polynomial loses the shape of data. In Figures 10 and 12, the dataset of Table 4 is interpolated by Theorem 3 . The interpolated curve is lying above the line $y=x+2$ in Figures 10 and 12, signifying that the shape of the data is preserved. Figure 11 is a close view of Figure 10.

Example 4. The 2D data in Table 5 consists of only three points and it is lying above the line $y=0.5 x-1$. The curve generated by interpolation of this data using $C^{2}$-quintic Hermite polynomial (Farin, 2005) is shown in Figure 13. It is lying below the line $y=0.5 x-1$, contrasting the shape of data. The same data is interpolated by Theorem 3 in Figures 14 and 15 and it is clear that the $C^{2}$-rational quintic function preserved the shape of data.

\section{CONCLUSION}

An alternate $C^{2}$-rational interpolation scheme is proposed for $2 \mathrm{D}$ data. The approximation order of the rational quintic interpolant is $O\left(h_{i}^{3}\right)$. It has three free parameters. One of the free parameters is converted to a shape-preserving parameter by imposing shapepreserving constraints on it. The other two are free for shape refinement. A comparison of the proposed shapepreserving schemes to the existing ones reveals that it has increased the order of continuity without constraining the first and second order derivatives at knots. 


\section{REFERENCES}

Butt S. \& Brodlie K.W. (1993). Preserving positivity using piecewise cubic interpolation. Computers and Graphics 17(1): $55-64$.

Duan Q., Wang L. \& Twizell E.H. (2005). A new $C^{2}$ rational interpolation based on function values and constrained control of the interpolant curves. Applied Mathematics and Computation 161: 311 - 322 .

DOI: https://doi.org/10.1016/j.amc.2003.12.030

Duan Q., Zhang H., Zhang Y. \& Twizell E.H. (2007). Error estimation of a kind of rational spline. Journal of Computational and Applied Mathematics 20(1): 1 - 11 . DOI: https://doi.org/10.1016/j.cam.2005.12.007

Farin G. (2005). Curves and Surfaces for CAGD: A Practical Guide, $5^{\text {th }}$ edition. Morgan Kaufmann Publishers, USA.

Goodman T.N.T., Ong B.H. \& Unsworth K. (1991). Constrained interpolation using rational cubic splines, Proceedings of NURBS for Curve and Surface Design (ed. G. Farin), pp. $59-74$.

Hussain Y. (2006). Monitoring and evaluation of efluents pollution of five drains before entering the chenab river. MSc thesis, Department of Environment Science, Lahore College for Women University, Lahore, Pakistan.

Hussain M.Z. \& Sarfraz M. (2008). Positivity-preserving interpolation of positive data by rational cubics. Journal of Computational and Applied Mathematics 218: 446 - 458. DOI: https://doi.org/10.1016/j.cam.2007.05.023

Hussain M., Hussain M.Z. \& Cripps R.J. (2009). C² rational quintic function. Journal of Prime Research in Mathematics 5: $115-123$.

Hussain M.Z., Sarfraz M. \& Shaikh T.S. (2011). Shapepreserving rational cubic spline for positive and convex data. Egyptian Informatics Journal 12: 231 - 236. DOI: https://doi.org/10.1016/j.eij.2011.10.002

Hussain M.Z., Hussain M. \& Waseem A. (2014). Shapepreserving trigonometric functions. Computational and Applied Mathematics 33(2): 411 - 431.

DOI: https://doi.org/10.1007/s40314-013-0071-1

Lamberti P. \& Manni C. (2001). Shape-preserving functional interpolation via parametric cubics. Numerical Algorithms 28: $229-254$.

DOI: https://doi.org/10.1023/A:1014011303076

Lettieri D., Manni C. \& Speleers H. (2014). Piecewise rational quantic shape-preserving interpolation with high smoothness. Jaen Journal on Approximation 6(2): 233 280.

Sarfraz M., Hussain M.Z. \& Hussain M. (2013). Modeling rational spline for visualization of shaped data. Journal of Numerical Mathematics 21(1): 63 - 87. DOI: https://doi.org/10.1515/jnum-2013-0003

Schmidt J.W. \& Hess W. (1988). Positivity of cubic polynomial on intervals and positive spline interpolation. BIT Numerical Mathematics 28: 340 - 352. DOI: https://doi.org/10.1007/BF01934097

Schumaker L.L. (2007). Spline Functions: Basic Theory, $3^{\text {rd }}$ edition. Cambridge University Press, Cambridge, UK. DOI: https://doi.org/10.1017/CBO9780511618994

Wang Q. \& Tan J. (2004). Rational quartic spline involving shape parameters. Journal of Information and Computing Science 1(1): 127 - 130.

Yong-juan P. \& Guo-jin W. (2007). Convexity-preserving interpolation of trigonometric polynomial curves with shape parameter. Journal of Zhejiang University SCIENCE A 8(8): 1199 - 1209.

DOI: https://doi.org/10.1631/jzus.2007.A1199 


\section{Appendix}

Table I: Numerical results of Figure 4

\begin{tabular}{cccccc}
\hline$i$ & 1 & 2 & 3 & 4 & 5 \\
\hline$d_{i}$ & -0.4215 & 0.2402 & 0.2775 & 0.0125 & -0.1393 \\
$D_{i}$ & 0.0456 & 0.0213 & -0.0600 & -0.0814 & 0.0426 \\
$\alpha_{i}$ & 0.005 & 0.005 & 0.005 & 0.005 & - \\
$\beta_{i}$ & 1.6124 & 1.0023 & 1.0002 & 1.0009 & - \\
$\gamma_{i}$ & 0.005 & 0.005 & 0.005 & 0.005 & - \\
$k_{i}$ & 62.8267 & 1.1850 & 1.0051 & 1.000 & - \\
\hline
\end{tabular}

Table III: Numerical results of Figure 7

\begin{tabular}{ccccccc}
\hline$i$ & 1 & 2 & 3 & 4 & 5 & 6 \\
\hline$d_{i}$ & -0.6063 & 0.1510 & 0.1425 & -0.0975 & -0.0050 & 0.0750 \\
$D_{i}$ & 0.1424 & 0.0357 & -0.0421 & -0.0169 & 0.0431 & 0.0369 \\
$\alpha_{i}$ & 0.0040 & 0.0040 & 0.0040 & 0.0040 & 0.0040 & - \\
$\beta_{i}$ & 1.0263 & 1.0012 & 1.0000 & 1.0015 & 1.0012 & - \\
$\gamma_{i}$ & 0.0040 & 0.0040 & 0.0040 & 0.0040 & 0.0040 & - \\
$k_{i}$ & 3.7963 & 1.0905 & 1.0000 & 1.1083 & 1.0862 & - \\
\hline
\end{tabular}

Table V: Numerical results of Figure 10

\begin{tabular}{cccccc}
\hline$i$ & 1 & 2 & 3 & 4 & 5 \\
\hline$d_{i}$ & -4.6950 & -1.0882 & 1.0395 & 1.4528 & 2.6763 \\
$\mathrm{D}_{\mathrm{i}}$ & 1.0496 & 0.5134 & 0.0855 & 0.3288 & 0.7146 \\
$\alpha_{i}$ & 0.0007 & 0.0007 & 0.0007 & 0.0007 & - \\
$\beta_{i}$ & 1.0000 & 1.0062 & 1.0000 & 1.0000 & - \\
$\gamma_{i}$ & 0.0007 & 0.0007 & 0.0007 & 0.0007 & - \\
$k_{i}$ & 1.3651 & 6.9167 & 1.1969 & 1.1164 & - \\
\hline
\end{tabular}

Table VII: Numerical results of Figure 14

\begin{tabular}{cccc}
\hline$i$ & 1 & 2 & 3 \\
\hline$d_{i}$ & -6.7778 & 4.1111 & 8.7778 \\
$D_{i}$ & -3.8025 & 5.8765 & 10.0247 \\
$\alpha_{i}$ & 0.0300 & 0.0300 & - \\
$\beta_{i}$ & 1.0137 & 1.0000 & - \\
$\gamma_{i}$ & 0.0300 & 0.0300 & - \\
$k_{i}$ & 22.6667 & 1.3449 & - \\
\hline
\end{tabular}

Table II: Numerical results of Figure 5

\begin{tabular}{cccccc}
\hline$i$ & 1 & 2 & 3 & 4 & 5 \\
\hline$d_{i}$ & -0.4215 & 0.2402 & 0.2775 & 0.0125 & -0.1393 \\
$D_{i}$ & 0.0456 & 0.0213 & -0.0600 & -0.0814 & 0.0426 \\
$\alpha_{i}$ & 1.0000 & 1.0000 & 1.0000 & 1.0000 & - \\
$\beta_{i}$ & 123.4819 & 2.4051 & 1.1285 & 1.1781 & - \\
$\gamma_{i}$ & 3.0000 & 3.0000 & 3.0000 & 3.0000 & - \\
$k_{i}$ & 62.8267 & 1.1850 & 1.0051 & 1.000 & - \\
\hline
\end{tabular}

Table IV: Numerical results of Figure 8

\begin{tabular}{ccccccc}
\hline$i$ & 1 & 2 & 3 & 4 & 5 & 6 \\
\hline$d_{i}$ & -0.6063 & 0.1510 & 0.1425 & -0.0975 & -0.0050 & 0.0750 \\
$D_{i}$ & 0.1424 & 0.0357 & -0.0421 & -0.0169 & 0.0431 & 0.0369 \\
$\alpha_{i}$ & 2 & 2 & 2 & 2 & 2 & - \\
$\beta_{i}$ & 24.6042 & 2.2445 & 1.0000 & 1.5865 & 2.1905 & - \\
$\gamma_{i}$ & 5 & 5 & 5 & 5 & 5 & - \\
$k_{i}$ & 3.7963 & 1.0905 & 1.0000 & 1.1083 & 1.0862 & - \\
\hline
\end{tabular}

Table VI: Numerical results of Figure 12

\begin{tabular}{cccccc}
\hline$i$ & 1 & 2 & 3 & 4 & 5 \\
\hline$d_{i}$ & -4.6950 & -1.0882 & 1.0395 & 1.4528 & 2.6763 \\
$D_{i}$ & 1.0496 & 0.5134 & 0.0855 & 0.3288 & 0.7146 \\
$\alpha_{i}$ & 2 & 2 & 2 & 2 & - \\
$\beta_{i}$ & 1.0000 & 18.6747 & 1.0000 & 1.0000 & - \\
$\gamma_{i}$ & 10 & 10 & 10 & 10 & - \\
$k_{i}$ & 1.3651 & 6.9167 & 1.1969 & 1.1164 & - \\
\hline
\end{tabular}

Table VIII: Numerical results of Figure 15

\begin{tabular}{cccc}
\hline$i$ & 1 & 2 & 3 \\
\hline$d_{i}$ & -6.7778 & 4.1111 & 8.7778 \\
$D_{i}$ & -3.8025 & 5.8765 & 10.0247 \\
$\alpha_{i}$ & 2 & 2 & - \\
$\beta_{i}$ & 10.1026 & 1.0000 & - \\
$\gamma_{i}$ & 10 & 10 & - \\
$k_{i}$ & 22.6667 & 1.3449 & - \\
\hline
\end{tabular}

